\title{
30 FRASES CONTABLES SOBRE ACTIVOS INTANGIBLES (COMENTADAS)
}

\author{
30 INTANGIBLE ASSETS ACCOUNTING PHRASES (ANNOTATED) \\ Román Arturo Valdivia Ramos* \\ Docente Asociado de la Facultad de Ciencias Contables
}

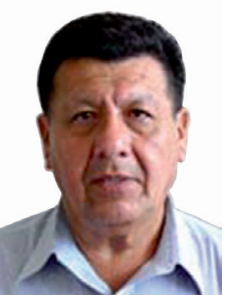

Universidad Nacional Mayor de San Marcos-UNMSM / Lima-Perú

[Recepción: Julio de 2013/ Conformidad: Agosto 2013]

\section{RESUMEN}

Este trabajo de investigación, fundamentalmente trata sobre los activos intangibles de una empresa y su relación, con la Ciencia Contable. La contabilidad tradicional aún no es capaz de registrar los activos intangibles; por consiguiente, se hace necesario un cambio drástico y sustancial en sus procedimientos y normatividad. Cada día que pasa, para una empresa u organización, los activos intangibles suben o aumentan su valor; en cambio, los activos tangibles a medida que pasa el tiempo, lo van perdiendo. Hay una relación inversa.

Por otro lado, la contabilidad es dinámica, cambiante, por lo tanto, debe adecuarse a las necesidades y requerimientos de la empresa, inclusive aún a costa de cambiar paradigmas fuertemente enraizados en muchos contadores, que en lugar de interpretar el negocio, están "más interesados en perpetuar una práctica que les deje algunos dividendos". Se han olvidado que la tendencia actual es hacer una contabilidad totalmente distinta, tanto de forma como de fondo.

Somos optimistas, por lo tanto, estamos convencidos que la contabilidad actual (no la del futuro), va a crear modelos y sistemas que permitan registrar y contabilizar los activos intangibles.

\section{Palabras Clave:}

Activos intangibles, contabilidad actual, valor razonable, nuevos tipos de contabilidad, nuevo paradigma contable.

\begin{abstract}
This research mainly discusses the intangible assets of a company and its relationship with the Accounting Science. Traditional accounting is not yet able to record intangible assets, therefore, a drastic and substantial change in its procedures and regulations is necessary. Every day that passes, for a company or organization, intangible assets or increase its value up, whereas tangible as time goes on assets, are lost. There is an inverse relationship.

On the other hand, accounting is dynamic, changing, therefore, must be adapted to the needs and requirements of the company, even at the cost of changing paradigms deeply rooted in many counters, instead of interpreting the business, are "more interested in perpetuating a practice that leaves them some dividends. "They have forgotten that the current trend is to make a completely different accounts of both form and substance.

We are optimistic, therefore, we are convinced that the current accounting (not the future), will create models and systems to record and accounting for intangible assets.
\end{abstract}

\section{Keywords:}

Intangible assets, current accounts, fair value accounting new types, new accounting paradigm.

\footnotetext{
* Licenciado en Administración. Ingeniero Industrial. Docente Investigador de la Facultad de Ciencias Contables.

Email: arturo.valdivia.ramos@gmail.com
} 


\section{INTRODUCCIÓN}

La ciencia contable, poco o casi nada a lo expresado sobre los activos intangibles y, es que la contabilidad no fue creada para registrar inmateriales o cosas abstractas, sino para registrar lo tangible, lo material, lo concreto, lo que tiene evidencia. Como sabemos, los intangibles hoy en día, tienen más valor que los tangibles en una empresa u organización, pero no se ven, ni se pueden tocar, pero existen en toda organización.

El propósito del estudio es brindar información, señalando algunas frases contables referidas a los activos intangibles, que datan desde el año 1964 a la fecha. Hermanson (1964), fue el primero en escribir sobre los activos intangibles, cuando en su agenda de investigación se refirió a la contabilización de los Recursos Humanos y de esto, solo han pasado menos de 50 años.

Por lo tanto, escribir sobre este tema, se hace necesario en el campo contable. Lo que queremos y deseamos, es despertar en las personas que trabajan en esta noble profesión; el interés por aquellos aspectos que se refieren a la marca, el modelo, la identidad, el prestigio, la reputación, el Know - How, los valores, entre miles de otros intangibles.

¿Acaso, esto no es lo más importante para cualquier empresa?

¡Y si es importante, lo es también para la Contabilidad!

¡Te reto para que lo registres y contabilices!

\section{FRASES CONTABLES SOBRE ACTIVOS IN- TANGIBLES}

\section{Frase contable}

Guilding and Pike 1990

Aunque no parece haber un método generalmente aceptado para la valoración de las Marcas, hay varios enfoques fundamentales que podrían competir por la aceptación general:
a. Coste histórico
b. Valor de mercado
c. Precio pagado en exceso
d. Valor actual neto
e. Valoración de la fortaleza de la marca

\section{Comentario}

No existe en la actualidad un método, modelo o sistema único que permita valorar o medir la Marca.

\section{Frase Contable}

Los resultados del estudio empírico llevado a cabo por Kim y Chung/1997, sobre la base de una muestra de empresas automovilísticas de EE.UU. y Japón, indican que existen activos intangibles específicos de cada país (fundamentalmente la popularidad de las Marcas), que están significativamente relacionadas con la cuota de mercado de la firma.

\section{Comentario}

La marca es uno de los principales activos intangibles de toda empresa, por lo tanto, deben ser registrados contablemente, pero no con el método actual de la contabilidad, sino con modelos que se acerquen más a la realidad. Debemos abandonar prácticas obsoletas e incorporar aquellas que son efectivas en las nuevas circunstancias.

\section{Frase contable}

Algunos "textos" de Contabilidad (usados en algunas universidades), siguen explicando y enseñando solo teneduría de activos, pasivos y patrimonio, como si luego de Luca Paciolo no se hubiera realizado aporte innovador (Samuel A. Mantilla B./2004).

\section{Comentario}

Las tendencias actuales señalan que la Contabilidad de hoy, es totalmente diferente a la Contabilidad tradicional y será más diferente en el futuro, tanto en la forma como de fondo.

Podríamos decir que, es otra Contabilidad que ya replantea nuevos principios y postulados, incluso paradigmas.

\section{Frase contable}

Hace décadas, la contabilización de los recursos humanos apareció en la Agenda de la investigación con el trabajo de Hermanson / 1964. 


\section{Comentario}

Se han cumplido casi 50 años de haber aparecido la Contabilidad de los Recursos Humanos en el mundo; por lo tanto, no debe ser considerada como algo nuevo o novedoso. Su medio siglo de existencia, indica la preocupación en esos tiempos, de la enorme importancia que tenía, tiene y tendrá el recurso humano, que en la actualidad ha sido cambiado por capital humano.

\section{Frase contable}

A finales de los años 60 y principios de los 70 , se convirtió en el centro de un fructífero debate que estaba fundamentalmente motivado por la cuestión de silos EE.FF. deberían incluir información sobre el valor de los recursos humanos. Poco después, al final de los 70, el interés en la Contabilidad de los recursos humanos (Brummet, Flamholtz and Pyle / 1968), disminuyó significativamente, como consecuencia de las implicaciones prácticas de la investigación publicada hasta el momento.

\section{Comentario}

Posteriormente, los recursos humanos cambiaron para convertirse en Capital Humano, es decir, en el capital más importante de toda empresa o institución, dejando de lado incluso al capital dinero, maquinarias y equipos, infraestructura, etc.

\section{Frase contable}

Los primeros años de la década de los 90, han sido testigos de algunos intentos por parte de la comunidad académica de revivir el interés en la contabilización de los recursos humanos, ya que el valor de los empleados se considera como un determinante clave del éxito de las compañías en sectores basados en el conocimiento (Postrel/1996).

\section{Comentario}

La contabilidad del conocimiento, se empieza a estudiar en los primeros años de la década del 2000.

Por primera vez en el Perú, en el Doctorado de Ciencias Contables y Empresariales de la Facultad de Ciencias Contables - Universidad Nacional Mayor de San Marcos, se enseñó la asignatura Contabilidad del
Conocimiento, en el año 2003 y el docente a cargo del dictado fue el Dr. Ernesto Polar Falcón.

\section{Frase contable}

De acuerdo con Parkes/ 1997, no reflejar el capital intelectual en los EE.FF. de las empresas de servicio basadas en el conocimiento, implica olvidarse de uno de sus activos fundamentales.

\section{Comentario}

Se refiere al hecho de que los EE.FF. son incompletos al no poder reflejar activos intangibles como el Capital Intelectual, que está constituido por los siguientes capitales:
a. Capital humano
b. Capital estructural
c. Capital relacional

\section{Frase contable}

Los activos intangibles son parte importante del valor de mercado de las empresas y organizaciones en general, su análisis corresponde a la necesidad de la contabilidad tradicional, que no proporciona la información suficiente en relación con la medición y valuación de dichos recursos (@Gerencia.com Activos Intangibles).

\section{Comentario}

Efectivamente los activos intangibles son los que dan valor agregado a los productos y/o servicios. Lo que sucede es que la Contabilidad no tiene aún los métodos y procedimientos para registrarlos contablemente.

La contabilidad tradicional, por lo tanto, es insuficiente para llevar a cabo el registro contable de los activos intangibles.

\section{Frase Contable}

En el desarrollo de la economía que hace énfasis en el conocimiento se ha destacado la importancia de los activos intangibles como elementos generadores de valor, teniendo en cuenta que usualmente se da mayor importancia a los recursos físicos y monetarios, sin tener presente que la identificación de los activos intangibles es una nueva realidad; la cual le 
proporciona al ente que los valora, mayor eficiencia yverdaderas fuentes de creación de valor (@Gerencia.com)

\section{Comentario}

La nueva contabilidad debe y está obligada a registrar los activos intangibles de una empresa, pues le otorga una fuente inagotable de valor y cada día que pasa, esta crece.

\section{Frase CONTABle}

Los activos intangibles están compuestos por derechos susceptibles de valor económico; identificables, tienen carácter no monetario y carecen de apariencia física (CEF-Contabilidad).

\section{Comentario}

La novedad que incorpora esta definición, está dada en que el activo intangible es identificable, lo que implica:

a. Que sean separables, es decir, pueden ser vendidos, cedidos o entregados a otros para su comercialización o intercambio.

b. Tienen derechos legales, es decir, pueden ser transferidos a otras empresas o instituciones.

\section{Frase contable}

El estudio de los intangibles, ha sido abordado de varios puntos de vista, ya sea como activos intangibles, activos del conocimiento y capital intelectual, todos son términos ampliamente divulgados. Activo intangible en la literatura contable, activo del conocimiento en la literatura económica y capital intelectual en la literatura de Dirección Estratégica de empresas (Reinier Hollander Sanhueza).

\section{Comentario}

Todos estos términos son sinónimos, es decir, son lo mismo y en esencia se refieren a las características no físicas (no es percibido por el tacto y gusto, es impalpable e imperceptible), que generan beneficios económicos futuros para la empresa u organización.

\section{Frase contable}

La incapacidad de la contabilidad de reflejar fielmente todos los hechos económicos, ya que muchos de ellos no son susceptibles de ser contabilizados por carecer de objetividad en cuanto al reconocimiento como a la valuación.

Si bien, la contabilidad pretende entregar información reflejando la posición financiera de la empresa, ésta no responde plenamente a los requerimientos de los usuarios (Reinier Hollander Sanhueza).

\section{Comentario}

El SFAC N ${ }^{\circ} 5$ (Statement of Financial Accounting Concepts)/FASB, establece que los EE.FF. no proporcionan información de todos los activos y pasivos de la empresa, sino que sólo refleja aquellos que cumplen ciertas condiciones.

La Contabilidad no puede registrar contablemente el conocimiento, la reputación, al cliente cautivo, entre otros intangibles.

\section{Frase CONTABLE}

Dado que el entorno económico cambia y que más empresas crean ventajas competitivas mediante su capital intelectual, la diferencia entre el valor de mercado de una empresa y el valor contable es cada vez más amplio (los Activos Intangibles: Limitaciones en la Contabilidad Financiera y oportunidades en la Contabilidad de Gestión de Michael Tayles/ Hull University Business School).

\section{Comentario}

Se debe encontrar un método fiable para medir los activos inteligentes y desarrollar indicadores que permiten una comprobación exacta.

\section{Frase contable}

En los círculos Contables - Financieros, cada vez está más aceptado que el valor de las empresas se base, principalmente, en los activos intangibles (Guthrie, 2001).

\section{Comentario}

Los contadores reconocen que los activos intangibles son los que proporcionan en mayor medida a la creación de valor para el dueño de la empresa, sin embargo, la actual normativa contable, aún no puede reflejar esta realidad. 


\section{Frase Contable}

Cuando se analizan los activos intangibles en un contexto de Capital Intelectual, el planteamiento básico es completamente diferente: su vida útil es indeterminada (puede ser muy corta o muy larga, depende del mercado y en el fondo se trata de un riesgo) y su valor aumenta con su uso, esto es, no pueden ser depreciables. Por lo tanto, los activos intangibles es igual a Capital Intelectual. De la misma manera, debe tener presente que no existen pasivos intangibles ¡Crisis absoluta para la partida doble! (Samuel A. Mantilla B./2004).

\section{Comentario}

La base del registro ya no está en la partida doble, sino en la base de los datos financieros y No financieros, mediciones cuantitativas y cualitativas, entre otros; que se orienten a las necesidades de cada cliente o usuario.

\section{Frase contable}

La contabilidad es el lenguaje de los negocios y dada la evolución de estos en las últimas épocas y sus tendencias más importantes, está claro el horizonte hacia donde tiene que cambiar, y lo está haciendo (Samuel A. Mantilla B./2004).

\section{Comentario}

No existe ninguna duda que la contabilidad tradicional, está dando paso a una nueva contabilidad más realista, más verdadera con el apoyo de las NIC y NIIF.

No olvidemos que la contabilidad es una ciencia dinámica que está en constante cambio y transformación.

\section{Frase contable}

Si el negocio, las organizaciones, la administración y el estilo gerencial de manejo cambian, entonces la Contabilidad, el control interno, el costo, la auditoría, también deben cambiar (Samuel A. Mantilla B./2004).

\section{Comentario}

Es obvio que los negocios cambiaron. Ahora son múltiples, complejos y veloces. El problema ya no es entre deudor y acreedor, ahora los problemas giran alrededor de agregar valor al cliente y al dueño de la empresa.

Las organizaciones, en estos tiempos son más horizontales que verticales, la administración ha cambiado radicalmente y el estilo general actual nada tiene que ver con el estilo gerencial de hace apenas 5 años atrás.

\section{Frase Contable}

Hoy las tecnologías de la información han abierto campos insospechados para el registro mismo (léase Contabilidad y Auditoría en tiempo real), para la agregación/desagregación de información (léase planes de cuentas y armonización contable), para la generación de múltiples reportes financieros (léase nueva información financiera) (Improving Business Reporting/AICPA-1997; Jenkins, Edmund).

\section{Comentario}

Incluso se ha llegado al corazón mismo del registro - contable: la partida doble que ya marca su obsolescencia y cede el paso a las partidas matriciales, existiendo incluso Contabilidad sin registro. Ante la nueva tecnología contable, la profesión se volvió otra y se tornó en imperativo replantear los planes de estudio y la formación de los contadores profesionales (Estándares Internacionales de Educación para contadores profesionales de la IFAC).

\section{Frase Contable}

Los tradicionales sistemas de contabilidad se organizaron alrededor del costo histórico como reflejo fidedigno de las transacciones de intercambio. La critica principal que hoy se les hace en su perspectiva pasada e interna, dado que el mundo cambió hacia una percepción futura (prospectiva) y del entorno (mercados).

El valor razonable lo reemplazó prácticamente por completo. Para llegar a ello, se han realizado diferentes esfuerzos orientados todos a mejorar la información histórica ajustándola, re-expresándola y actualizándola en el tiempo. El problema principal, sin lugar a dudas, radica en que la moneda funcional 
con la cual se valora la información contable está sujeta a demasiados cambios por efecto de inflación, devaluación y otros.

La crisis se abordó con la explosión de los costos: de reposición, valor de mercado, etc, que en el fondo no eran otra cosa que distintas maneras de expresar el costo histórico en el tiempo. Pero los negocios del presente van haciendo que ello no sea tal útil ni necesario como se creía. Lo que sucede es que la historia de los negocios ya no es pasada, sino que es presente y futura. Técnicamente, ello se denomina medición del desempeño financiero.

Sin lugar a dudas, la medición de la posición financiera ya no es suficiente. De ahí, se reitera, la emergencia del valor razonable. Igual sucedió con los Presupuestos; hoy se toman decisiones basadas en Sistemas de Contabilidad construidos sobre Base Cero: reflejan el negocio actual y como es hoy jincluyendo la valoración y el registro del riesgo! Ya no se acepta cambiar bases comprensivas de Contabilidad o estar cerca de los estándares internacionales, o tomados parcialmente. La adopción tiene que ser plena. Y ello implica empezar de nuevo (Base Cero) (Este es un asunto central en IFRS 1, Adopción por primera vez de los IFRS).

\section{Comentario}

Diversos autores han preferido referirse a la contabilidad por procesos, proyectos o actividades, a condición de que se cree valor (Management Accounting de Atkinson, Banker and Young / 1997).

La idea fundamental es que la contabilidad no debe registrar cosas del pasado. La Contabilidad es presente e incluso es más del futuro, porque debe servir para proyectar el negocio y decidir ahora, si está o no en capacidad de continuar todo lo andado.

\section{Frase contable}

Ahora se tiene mayor conciencia de que la empresa no está sola en el universo empresarial. Por ello, se insiste en que los EE.FF. de la empresa de alguna manera tienen que reflejar la relación de ésta en el contexto en que se mueve y, de manera especial, con el sector económico al que pertenecen y frente a la competencia. Por ello la insistencia globalizada de la armonización contable y de los EE.FF. comparativos (El asunto ya se veía venir desde 1998, IASC / Combinaciones de negocios).

\section{Comentario}

Pero algunos van más lejos aún, insisten en que los EE.FF. de la empresa, muestren el impacto de su relación con el exterior.

La discusión al respecto no ha llegado a una solución por los problemas derivados de lo ambiental (IFAC - FMAC / 1998).

Existen 3 propuestas principales:

a. Que los EE.FF. incluyan las cuentas ambientales.

b. Que los EE.FF. comprendan 2 partes: Financiera y No Financiera o ambiental

c. Que estén separados los EE.FF. y los EE. No FF. O EE. AA. (Estados Ambientales) (Gray, Bebbington and Walters / 1993, Accounting for the Environment).

\section{Frase Contable}

La contabilidad está transformándose hacia un nuevo enfoque o paradigma: de la Contabilidad de intercambio (Comercio) y la Contabilidad de Producción (Industria), se pasa ahora a la Contabilidad del Servicio (Producción, intercambio y agregación / generación de valor) (Anónimo).

\section{Comentario}

Esto se debe a la introducción de nuevas formas de comunicación, la electrónica, la informática, la computación, la mecatrónica y otros, que han llevado a las empresas a una nueva manera o forma de hacer negocios; y además, con nuevos estilos gerenciales, nunca antes visto. Todo esto, hace cambiar a la Contabilidad.

\section{Frase CONTABLE}

Si bien es cierto que, en el área de los negocios, el sistema más aceptado de medición del desempeño ha sido la Contabilidad, hoy está claro que ésta ha perdido la primacía como sistema único de medición de los negocios (Sutton, Steve G. / 1998).

128/ QVIPURAMAYOC | Vol. 21(40) 2013 


\section{Comentario}

En efecto, existen otros modos o formas de medir la performance o desempeño (rendimiento), más ajustados a la realidad, pues son más complejos e interrelacionados. Cuando los negocios utilizan exclusivamente mediciones cuantitativas o ratios financieros, sin aplicar otros medios de evaluación, como es el caso de las mediciones cualitativas; romper la comprensión natural que las personas tienen de "ver la manera de cómo funcionan los procesos".

\section{Frase CONTABLE}

La Contabilidad ha ido perdiendo buena parte de su eficacia práctica y ha ido cediendo terreno frente a otras respuestas técnicas, especialmente a:

- Valuación de los negocios

- Medición del desempeño

- Sistemas Integrados de Información
(Fernández, Pablo y Fitzgerald L., Johnston y otros / 1999).

\section{Comentario}

Esto no quiere decir que en la Contabilidad no haya habido avances y progresos. Todo lo contrario, pero aún falta mucho por recorrer. Lo que sucede es que el mundo actual es plural, abierto, interdisciplinario y las respuestas contables ya no son suficientes; es obligatorio insertarse en la red administrativa logística - operacional.

\section{Frase Contable}

Incluso se ha llegado a señalar que las campañas que prestan mayor atención a sus Stakeholders y menos a las medidas financieras mantendrán un futuro más sostenible (Wales, J, P. Smith, M. Wilson and A. Nokma/ 1999).

\section{Demanda de los diferentes Stakeholders}

\begin{tabular}{|c|c|l|}
\hline $\mathbf{N}^{\circ}$ & Stakeholders & \multicolumn{1}{|c|}{ Demanda } \\
\hline 1 & Accionistas & $\begin{array}{l}\text { Retorno financiero, valor de largo plazo por } \\
\text { acción, valor agregado }\end{array}$ \\
\hline 2 & Proveedores & Pago rápido, relaciones a largo plazo \\
\hline 3 & Acreedores & Merecedor (digno) de crédito, seguridad \\
\hline 4 & Empleados & $\begin{array}{l}\text { Pago justo, entrenamiento, satisfacción, salud, } \\
\text { seguridad social, igualdad de oportunidades }\end{array}$ \\
\hline 5 & Clientes & Suministro, calidad, valor del dinero \\
\hline 6 & Gobierno & Cumplimiento legal, responsabilidad social \\
\hline 7 & Competidores & Competencia justa \\
\hline 8 & Sociedad & Seguridad, contribución social, buen ciudadano \\
\hline 9 & Ambiente & Sostenibilidad, interés ambiental \\
\hline
\end{tabular}

(Samuel A. Mantilla B./2004)

\section{Comentario}

\section{Frase CONTABLE}

A menudo digo que cuando usted puede medir aquello sobre lo cual está hablando y lo expresa en números, usted sabe algo sobre ello; y cuando usted no lo puede medir, cuando no lo puede expresar en números, su conocimiento es escaso e insatisfactorio (William Thompson / Lord Kelvin, 1886).

\section{Comentario}

Se refiere a que todo es susceptible de ser medido, cuantificado, valorado o valuado. Si el resultado se lleva a números, mucho mejor, desde luego, sin desdeñar la medición cualitativa. Recordemos la frase de Peter F. Drucker: No se puede dirigir lo que no se mide. 


\section{Frase contable}

Los modelos contables estándar fueron diseñados para informar a la administración de la compañía y a los stakeholders sobre los inventarios y flujos de valor-valor que se podía atribuir a lugares, períodos de tiempo, productos, clientes y actividades. La mayoría de esos eran cuantificables y estaban sujetos a los principios y prácticas generalmente aceptados de contabilidad (GAAP).

\section{Comentario}

Eso fue en el antaño, hoy en día las cosas son diferentes y todavía la contabilidad actual aún no está en capacidad de contabilizar los activos intangibles, se requerirá de nuevos conceptos y prácticas de contabilidad para llevarlo a cabo.

\section{Frase Contable}

A manera de ejemplo, Toyota, la compañía de automóviles de mayor éxito en el mundo, tiene un extenso sistema de medición en sus plantas de producción, pero no usa ningún sistema estándar de contabilidad para centralizar el control administrativo. Toda su filosofía de medición se concentra en medir y evaluar para aprender Toyota, tiene un excelente sistema de contabilidad, pero no utiliza su información para tomar las divisiones de día a día sobre las operaciones. Su toma de decisiones se guía por una conciencia y sensibilidad al flujo de trabajo, hondamente arraigadas. No necesitan medidas contables para saber si un determinado proceso de trabajo o material no es adecuado, lo advierten inmediatamente. (Toyota).

\section{Comentario}

Si se utiliza la contabilidad tradicional, nada se consigue, en este caso, se vuelve obsoleta. En cambio, si la contabilidad actual adopta nuevas formas o maneras de registrar, las cosas cambiarán drásticamente.

\section{Frase Contable}

Hoy está claro que es necesario: Diferenciar entre Contabilidad, Contabilidades, Sistemas de Información Contable e Información para la generación de valor (Mantilla B. Samuel / 2004).

\section{Comentario}

Todos estos conceptos son diferentes, sin embargo, muchos aún tienden a considerarlos como sinónimos. Se debe entender claramente que una cosa es llevar una Contabilidad Tradicional y otra cosa es llevar diferentes tipos de Contabilidades que se adecúan o se amoldan a las necesidades y requerimientos del cliente.

\section{FRASE CONTABLE}

Las mediciones No Financieras son más importantes y de mayor valor que las mediciones Financieras. Sin embargo, debemos entender que ambas mediciones, siempre deben in juntas. Son como el cara y sello de una moneda (Arturo Valdivia Ramos, 2010).

\section{Comentario}

Las mediciones No Financieras son múltiples y diversas, sobresaliendo las siguientes:
a. Marca
b. Cliente
c. Servicio
d. Experiencia
e. Reputación
f. Cultura Corporativa
g. Políticas
h. Comunicaciones
i. Clima Laboral
j. Medio Ambiente, entre miles de otros.

\section{Frase Contable}

En un futuro cercano, el problema ya no será contar sino medir. Los problemas contables que se vienen, serán registrados a valor razonable o valor de mercado (como quiera llamársele), nunca más a valor o costo histórico (Arturo Valdivia Ramos /2011).

\section{Comentario}

El mundo empresarial camina rápidamente. Si el entorno empresarial cambia, la Contabilidad también cambia, así de simple. Y esto, se debe al cambio en el Paradigma o modo de pensar. Lo que antes era bueno, ahora no lo es y tampoco lo será para mañana.

130/ QVIPURAMAYOC | Vol. 21(40) 2013 


\section{CONCLUSIONES}

1. Los problemas del registro contable, hoy ya no existen: están todos resueltos y de manera eficiente, según lo han señalado por Peter F. Drucker y Robert Kaplan.

2. Ahora los problemas del registro contable giran alrededor de la medición de los Activos Intangibles.

3. Algunos piensan que la Contabilidad no debe cambiar, que debe ser la misma de siempre. Son ellos los que atrasan el futuro de la ciencia contable. Felizmente, la Contabilidad es una ciencia dinámica, activa que está en constante transformación o cambio a la par del "ritmo incesante de los negocios".

4. La contabilidad actual es y será totalmente diferente o distinta a la contabilidad tradicional, tanto en forma y en fondo.

5. Si la organización cambia, entonces la contabilidad también cambia.

6. La marca, el cliente, el mercado, la experiencia, la reputación y otros activos intangibles siempre valen más que la infraestructura, el producto, las maquinarias y equipos, los insumos, los materiales y otros activos tangibles.

7. Las tecnologías de la información han informatizado la contabilidad, llevándola a tiempo real y a la generación de múltiples reportes financieros.

8. El costo histórico ha sido cambiado y/o reemplazado para siempre por el Valor Razonable o Valor de Mercado.

9. Han aparecido nuevas contabilidades, como: Contabilidad Just in Time, Contabilidad del Conocimiento, Contabilidad Social, Contabilidad Ambiental, Contabilidad del Servicio, Contabilidad del Riesgo, entre otros tipos de Contabilidad. Entonces, estamos frente a un nuevo Paradigma Contable.

\section{REFERENCIAS BIBLIOGRÁFICAS}

1. Guilding and Pike. (1990). Intangible marketing asset: A managerial accounting perspective. Accounting and business research, winter.
2. Kim y Chung. (1997). Conference development \& Marketing manage.

3. Hermanson, R. H. (1964). Accounting for $\mathrm{Hu}-$ man Assets Occacional Paper 14.

4. Brummet, Flamholtz and Pyle. (1968). Human Capital Accounting.

5. Postrel. (1996). Virginia. The Future and its enemies/The susbtance of style.

6. Parkes. (1997). Psyclioanalitic culture.

7. @Gerence.com (1993) Activos Intangibles.

8. Centro de Estudios Financieros/España - Contabilidad.

9. Reinier Hollander Sanhueza. (2003) Modelo de Valoración de Activos Intangibles.

10. SFAC $\mathrm{N}^{\circ} 5$ Statements of Financial Accounting Concepts. (1984). Recognition and measurement in finacial statements of business.

11. Michael Tayles / Hull University Business School. (2008) Capital Intellectual.

12. Guthrie. (2001). Contexts for engagement and motivation.

13. Normas Internacionales de Contabilidad. (1995 y 2001).

14. Normas Internacionales de Información Financiera. (1995 y 2001).

15. Jenkins, Edmund. (1997), Improving Business Reporting. AICPA . Information innovation intangible economic.

16. IFRS 1. (Creación 2008, Aplicación 2009). FIRS - Time adoption of international financial reporting standars.

17. Banker and Young. (1997) Management Accounting de Atkinson,

18. IASC. (1998). Inter-agenncy standing conmmitee.

19. IFAC-FMAC. (1998). International Federation of Automatic Control.

20. Gray, Bebbington ton and Walters / Accounting for the Environment (1993). Can we reconcile finance with nature? 
21. Sutton, Steve G. (1998). Southern leaue.

22. Fernandez, Pablo y Fitzgerald L., Johnston y otros (1999). Valuación de empresas/Performance measurement in service business.

23. Wales, J, P. Smith, M. Wilson, and A. Nokma (1999). Stakeholder Enterprise System for Accountability, Measurement and Evaluation.

24. Samuel A. Mantilla B. (2004). Contabilidad intelectual \& Contabilidad del conocimiento.
25. William Thompson, Lord Kelvin. (1886). Frase Empresarial.

26. Peter F. Drucker. (1995). Sociedad del Conocimiento.

27. GAAP. (2005). Generally Acepted Accounting Principles.

28. Arturo Valdivia Ramos. (2012) Más allá de la NIC 38. Una mirada al futuro de los activos intangibles. 